\title{
The Newest Getter Technologies: Materials, Processes, Equipment
}

\author{
Konstantin Chuntonov ${ }^{1}$, Janez Setina², Gary Douglass ${ }^{3}$ \\ ${ }^{1}$ NanoShell Consulting, Nitzanim, Migdal Haemek, Israel \\ ${ }^{2}$ Institute of Metals and Technology, Ljubljana, Slovenia \\ ${ }^{3}$ Agile Chemistry, Inc., Elmhurst, USA \\ Email: konstantin@chuntonov.com
}

Received 31 August 2015; accepted 21 September 2015; published 24 September 2015

Copyright (C) 2015 by authors and Scientific Research Publishing Inc.

This work is licensed under the Creative Commons Attribution International License (CC BY). http://creativecommons.org/licenses/by/4.0/

(c) () Op Den Access

\section{Abstract}

The efficiency of sorption purification of gases, as measured by an improvement in product quality and/or lowering of its cost, can be significantly increased via simple solutions: the substitution of current getter technology with reactive getters; and stimulation of the material in the sorption process using mechanochemical methods instead of heating or cooling. These ideas were embodied by the authors in new sorption apparatuses and devices such as mechanochemical sorption apparatuses for production of ultra pure gases, improved gas purifiers with reactive sorbent for production of pure and high purity gases and, finally, fluidized bed columns for mass production of pure and high purity gases.

\section{Keywords}

\section{Reactive Getters, Gas Purifiers, Fluid Bed Column, Mechanochemistry}

\section{Introduction}

Big changes are brewing in the field of getter technologies: materials of more reactive nature (alloys of alkalialkaline-earth and some rare-earth metals) claim the place of getters containing transition metals. These new gas sorbents do not need heating or cooling; they are more economical to use in production, more convenient in their operation and much more effective in sorption. These new materials outperform the current getter materials by orders of magnitude in sorption capacity [1] [2].

Reactive getters can be used in place of all structural forms of sorption materials, such as films [3]-[14], porous bodies and composites [15]-[22], and powders [23]-[28], i.e. everything that can be used in purification processes for flow gases, for capturing of moisture in multilayer OLED films, for capturing of residual gases in 
vacuum chambers, etc. It was found that, in the most common getter applications, the powder form of reactive alloy was preferable for economic and sorption reasons. Therefore, special efforts were made in recent years which were focused on solving the problem of reactive getter powders.

The result of the research and development which was performed was the creation of an entire family of new technologies united by a common sorption agent and represented currently by sorption columns of three types: mechanochemical sorption apparatuses [28], gas purifiers using reactive sorbent and sorption columns of fluidized bed type [29]. This new equipment expands our practical possibilities, both in the studies of sorption processes and in their usage. The objective of this overview is to provide application specialists with the first knowledge about how the new technologies work and what can be expected from them.

\section{Materials-Reactive Metal Powder}

In the new technologies the gas impurity sorbents are alloys of reactive metals which sorb gases at room temperature. This is done by the growth of layers of chemical compounds on metallic surfaces. The choice of these types of new getter materials is easy to understand when they are compared to the sorption mechanism of the best known metal gas sorbents currently in use.

Figure 1 shows three types of sorption behavior of the metals which are used in getter technologies [2] [30][33]. Here $Q$ is the amount of gas sorbed by a unit surface area by the moment of time $t$.

Curve 1 describes the sorption law at $\mathrm{T}_{\text {room }}$ for transition metals like $\mathrm{Ti}, \mathrm{V}, \mathrm{Zr}, \mathrm{Ni}$, Fe, etc., which serve the material basis of modern getter products. It is seen that sorption stops at $t=t_{\mathrm{p}}$, when the surface is saturated with adatoms of gas. The ultimate value of $Q_{1}^{*}=k_{1} h_{\mathrm{p}} x_{1}$, where $k_{1}$ is the conversion factor and $x_{1}$ is concentration of gases in the passivated layer of thickness $h_{\mathrm{p}}$ answers this passivated state. The value of $h_{\mathrm{p}}$ is close to the thickness of a monoatomic layer.

Curve 2 describes the case of absorption: gases dissolve in the volume of the getter material. The value $Q$ at absorption asymptotically approximates the value of $Q_{2}^{*}$, which is limited by the maximal solubility of gas in the metals of the given class. $\mathrm{Ti}, \mathrm{V}, \mathrm{Nb}$ and $\mathrm{Zr}$ behave in this way towards hydrogen at $\mathrm{T}_{\text {room }}$, but for sorption of other gases these metals require heating. The ultimate value of specific sorption capacity at absorption can be found from the expression $Q_{2}^{*}=k_{1} h(K p)^{1 / 2}$, where $h$ is typical sorption size of the getter body (the thickness of the film, the radius of a spherical particle or a cylindrical needle, etc.), $K$ is an equilibrium constant and $p$ is partial pressure of gas molecules $Y_{2}$ above the getter.

Curves 3 and 4 refer to reactive metals which follow parabolic law $Q_{3}=k_{3} t^{1 / 2}$ (curve 3) or linear law $Q_{4}=$ $k_{4} t$ (curve 4). This is the case of alkali, alkaline-earth and some rare-earth metals, which sorb target gases continuously to completion, until the entire material is consumed, as the result of the reaction $\mathrm{Me}+\mathrm{Y}=\mathrm{MeY}$, where Me is metal, $\mathrm{Y}$ is sorbed gas, and $\mathrm{MeY}$ is the solid product of the reaction. Nitrogen sorption by barium

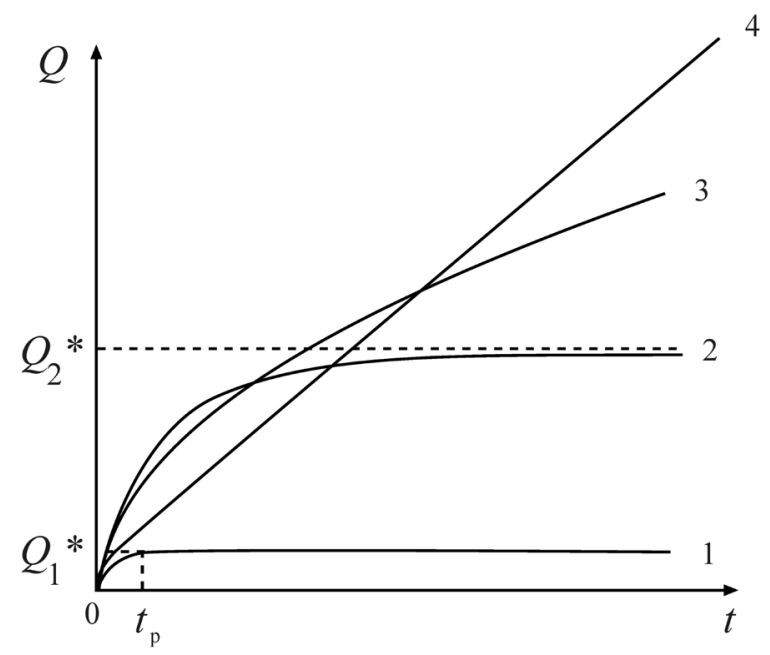

Figure 1. Sorption curves $Q(t)$ and getter classes: 1: adsorption, 2: absorption, 3 and 4: chemical reactions with the frontal movement according to the parabolic or linear law correspondingly. 
films [5] can serve as a convincing illustration of a parabolic law; and oxygen sorption by lithium films [10] as an illustration of a linear law.

For the ultimate values of sorption capacities of reactive metals we can write: $Q_{3}^{*}=Q_{4}^{*}=k_{1} h x_{3}$, where $x_{3}$ is the concentration of gas in MeY. Taking into consideration that $h_{p} \ll h$ and that the ultimate solubility of gas in metal at $\mathrm{T}_{\text {room }}$ is much lower than the concentration of this gas in the compound $\mathrm{MeY}$, we come as the result to the correlation of the ultimate values

$$
Q_{4}^{*}=Q_{3}^{*}>Q_{2}^{*} \gg Q_{1}^{*},
$$

from which it is seen that at room temperature the sorption capacities of reactive metals $\left(Q_{4}^{*}\right.$ and $\left.Q_{3}^{*}\right)$ are by orders of magnitude higher than those of transition metals $\left(Q_{1}^{*}\right)$ and their capacities are also higher than in the case of heating of the latter $\left(Q_{2}^{*}\right)$.

The process of gas purification in flow apparatuses depends not only on the sorption capacity of the getter material but also on the sorption rate, which limits the allowable flow rate. For any sorption column the sorption rate can be written down as $J=(\mathrm{d} Q / \mathrm{d} t) S$, where $\mathrm{d} Q / \mathrm{d} t$ is the specific sorption rate of the getter material and $S$ is the surface area of this material available for target gases. The larger the sorbing surface area $S$ the higher the sorption rate $J$; and this fact made us look for powder solutions, as it is powders which yield the maximal values of specific surface area. This explains why in our technologies the preference is given to reactive metals and why we prefer them in powder form.

The comparative sorption analogue of reactive powders from the side of the traditional getter materials are loose powders of transition metals or sintered powder materials on the basis of these metals in the form of pellets or high porous thick films [34] [35]. Figure 2 and Figure 3 show the sorption characteristics of three reactive alloys, intermetallic compounds of $\mathrm{Ba}_{8} \mathrm{Ga}_{7}$ and LiGa as well as eutectic alloy of $\mathrm{Ba}-20$ at\% In, these are compared to the established products of SAES Getters Inc. Measurements of the sorption properties were performed by dynamic flow method [36] [37], where an apparatus with a disintegrator [28], which allows mechanical milling of a reactive ingot in UHV conditions, served as a test chamber.

Figure 2 shows the sorption curves of nitrogen, which is one of the most difficult target gases to remove. As it can be seen at room temperature the sorption capacity of high porous getter film St. 122 [38] is approximately 20 times lower than that of the powder LiGa and approximately 500 times lower than that of the powder $\mathrm{Ba}-20$ at\% In. The mass of the reactive powder in each of the cases was equal to $0.12 \mathrm{~g}$ and the particle size was in the range of from $~ 50$ to 250 microns. Double-sided getter St. 122 had dimensions $2 \mathrm{~cm} \times 3 \mathrm{~cm}$ with getter mass $0.24 \mathrm{~g}$. It is quite clear that if in reactive alloys gallium or indium (which do not participate in nitrogen sorption) are replaced with a reactive metal, the sorption capacity of the getter powder will grow accordingly.

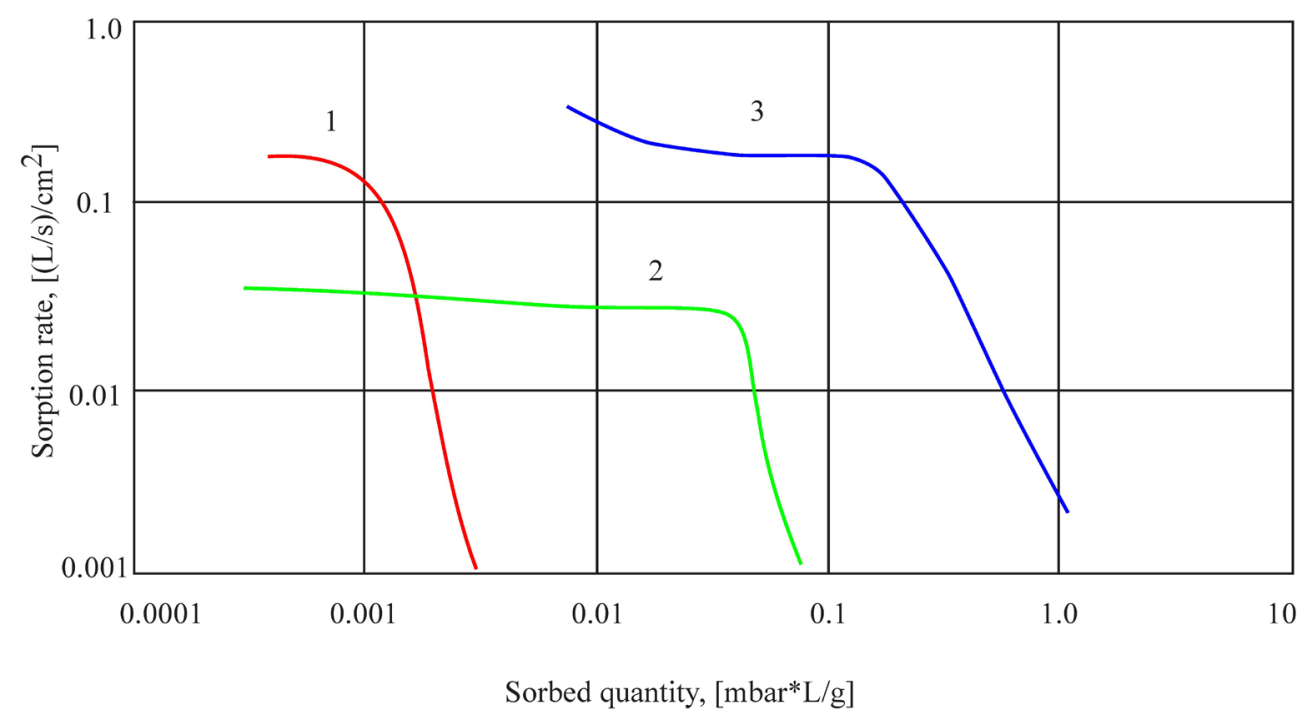

Figure 2. Sorption of nitrogen at room temperature: 1 : St. 122 activated at $450^{\circ} \mathrm{C}$ in vacuum $5 \times 10^{-7}$ mbar 1 hour; 2: powder of LiGa tested immediately after milling. 3: powder Ba-20 at\% In tested 24 hours after milling. 


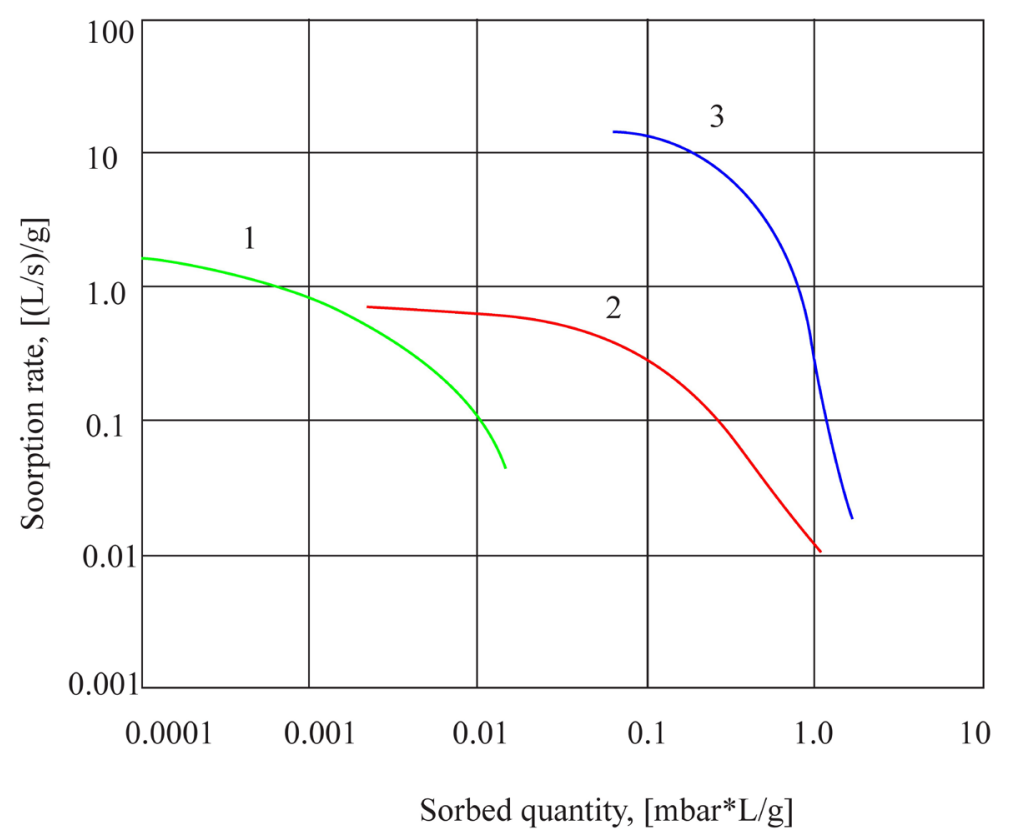

Figure 3. Sorption of oxygen and carbon oxides at room temperature: 1: St. 707 activated at $500^{\circ} \mathrm{C}$ for 10 minutes, test gas $\mathrm{CO}$ [39]; 2: powder $\mathrm{Ba}_{8} \mathrm{Ga}_{7}$ tested 340 hours after milling, test gas $\mathrm{O}_{2} ; 3$ : Ba-20 at\% In tested 24 hours after milling, test gas $\mathrm{CO}_{2}$.

Sorption characteristics of powders St. 707 as well as reactive powders of $\mathrm{Ba}-20$ at $\%$ In and $\mathrm{Ba}_{8} \mathrm{Ga}_{7}$ are given in Figure 3. Oxygen and carbon oxides, which are very similar in their sorption behavior, were used as test gases. The mass of the powder of St. 707 was equal to $0.2 \mathrm{~g}$ and the particle size was $40-128$ micron [39]; the mass of each of the reactive powders was $0.25 \mathrm{~g}$ and the particle size was the same as in the case of the tests with nitrogen. According to the experimental data (Figure 3 ) the specific sorption capacity of the reactive materials towards oxygen and oxygen containing gases is on average approximately two orders of magnitude higher than that of the current getters.

The results obtained look impressive as the 100 -fold advantage in sorption capacity immediately opens ways to industrial applications for reactive powders. With that said, the evaluation of the obtained values of sorption capacity of reactive metals shows that these values are far from the theoretical limit and that decreasing the average particle size to $\sim 10$ micron will allow an increase in the sorption capacity by 15 - 20 times more. Naturally, this fact identifies the need to improve the methods of the mechanical milling of reactive alloys. The solution can be reached by enhanced design or mechanical techniques and by influencing the structure of the ingot during the growth process.

We will now move from the materials overview to a comparative review of the sorption columns and the processes, which take place within them. Before we begin, let us remind the reader that, in contrast to the previous methods of gettering gases, the new technology does not require heating or cooling of the gas sorbent; in addition, it is by many times more effective in sorption while being significantly more economical to achieve.

\section{Improved Gas Purifiers with Reactive Sorbent}

New gas purifiers of in-line type are intended for the production of pure and high pure gases within systems of small or medium capacity [29]. They work at $\mathrm{T}_{\text {room }}$, have a typical look and are similar to currently used equipment in their operation; however, they are more convenient in operation, are completely safe and, most importantly, provide a 100 -fold increase of sorption capacity within the same outside dimensions. The experimental results which were discussed in the previous chapter are primarily applicable to this type of gas purification equipment.

The design of the new gas purifier can be seen in Figure 4 [29]: its housing is formed by two blunted cones, which are put together along their common base forming divergent/convergent gas flow without "dead zones”. 


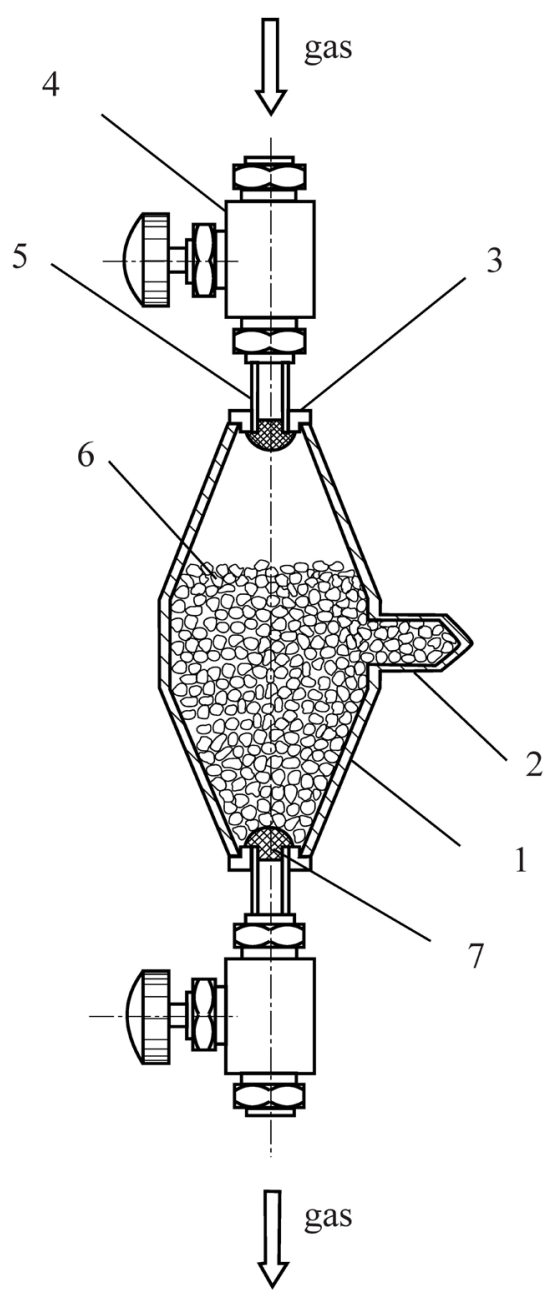

Figure 4. Gas purifier with reactive powder. 1: a steel housing with the remains of filling tube 2; 3: end ports; 4: a gas valve; 5: gas tubes; 6 : sorbent particles; 7: a filter.

Free pouring of powder particles minimizes the pressure drop and two gas valves on the ends of the device make it autonomous. This allows its installation or a change of its working position without loss of the gas sorbent and without leakage of the process gas to the atmosphere. In a new purifier, the presence of the space not occupied by the powder inside the housing is necessary because the volume of the particles will increase and occupy the remaining space during the gas sorption process.

The material bases for sorption powders are binary, ternary or multicomponent alloys, the concentration of reactive metals in which the range is from $50 \%$ to $100 \%$. It is under these conditions that gas sorption takes place according to the mechanism of chemical reactions and runs to completion. Among these alloys the preference is given to alloys of $\mathrm{Ba}-\mathrm{Al}, \mathrm{Ba}-\mathrm{Mg}$, as well as to alloys of $\mathrm{Ca}-\mathrm{Li}, \mathrm{Ca}-\mathrm{Mg}$ or $\mathrm{Ca}-\mathrm{Li}-\mathrm{Mg}$. It is not difficult to change the ratios of components in these alloys in order to create the required composition of getter material for purification of noble gases, nitrogen, hydrogen, etc.

The advantages of the improved gas purifiers are not solely limited to its sorption efficiency; they are attractive from the economical view point as well, due to the cheapness and availability of reactive metals. Market prices for reactive metals are by several times lower than for Ti, V and Zr. In the case of reactive powders the production costs to produce the end getter products from the initial metals are less expensive, by many times over. To illustrate, for the production of sintered powder porous materials, five technological operations are needed; in the case of reactive powders only two operations are needed. The creation of alloys of transition metals require high vacuum furnace with heating to $2000^{\circ} \mathrm{C}$ and higher, while reactive alloys are grown with the 
help of simple ampoule techniques with high rate in common tube furnaces at temperatures range from $600^{\circ} \mathrm{C}$ and lower. As a result, the cost for production of reactive powders is estimated to be approximately 10 times lower than for the production of the current getter materials.

If we define the total positive effect from the industry changeover to the new gas purifiers with a value expressed as $k=k_{1} \times k_{2}$, where $k_{1}$ is the number showing by how many times the sorption capacity of reactive metals is higher than the sorption capacity of the current sintered powder porous getters and the number $k_{2}$ is the number showing by how many times production costs of sintered powder porous getters are higher than that of the production of reactive powders, we come to $k=100 \times 10=1000$. This is a revolutionary result, especially taking into consideration that a decrease of the size of reactive particles to 10 micron is able to lead to the increase of the total effect by an additional order of magnitude.

\section{Mechanochemical Sorption Apparatuses}

These apparatuses were initially intended for the production of ultra pure gases [28]. They are new in principle equipment, which have no analogs in gas purification practice. Schematically such an apparatus is shown in Figure 5: The initial ingot loaded into disintegrator 2 and/or 2' and is milled directly in the atmosphere of purified gas $\mathrm{X}+\mathrm{Y}$, where $\mathrm{X}$ is the desired process gas, and $\mathrm{Y}$ is impurity. The gas, which enters the reactor through filter 4, goes then through a thin layer of as-made powder 12 and exits through filter 10.

The advantages of the mechanochemical method are described in detail in [27] [28], so here we will mention only the primary advantages, which are the controlled character of the purification process and the clear absence of the factors limiting the purity degree of the end product.

Controllability of the sorption process: For the first time in sorption purification technologies a feedback system 6 (Figure 5) allowed making this process controllable and this is done in a simple way: by regulating the feed rate of getter powder into the reaction zone. By changing the amount of the powder mass produced during a unit of time it is possible to achieve constant purity of the gas, which exits column at the desired rate of gas flow.

Control over the purification process is a new useful function, which not only facilitates the standardization of related production processes but is also capable of bringing reorganization in the gas supply systems. The users of ultra pure gases can now look forward to significant reduction or elimination of certain logistical issues associated with these gases by building mechanochemical apparatuses directly into their gas network.

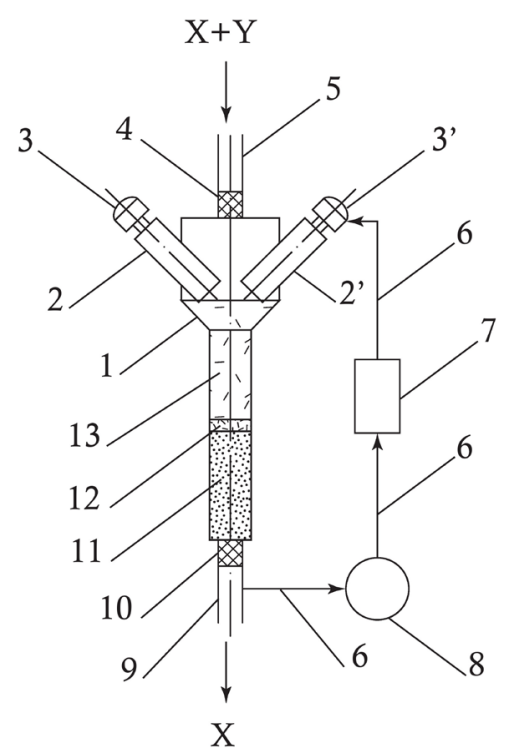

Figure 5. Mechanochemical sorption apparatus: 1: the impermeable to gas casing of the sorption column; 2 and 2': disintegrators; 3 and 3': outside actuators; 4: a filter; 5: an inlet; 6: a feedback line; 7: a controller; 8: a gas analyzer; 9: an outlet; 10: a filter; 11: used powder; 12: a layer of fresh powder; 13: falling particles. 
Extremely high purity of the output process gas: The estimations based on the data from gas permeability of stainless steels and in the data from partial pressure of the products MeY near ambient temperature show that mechanochemical purification of substances by reactive metals is able to decrease the content of active and low active impurities in them to $\sim 10^{-15} \%$. At present neither the gas industry nor the researchers have been able to set these kinds of goals for themselves as they have been absolutely unobtainable.

What is it that radically shifts the level of the achieved purity of the gas product in the mechanochemical method? Let us point out two factors. The first one is that in mechanochemical purification, which is carried out at ambient temperature, the sources of contamination which appear in getter technologies using the heating of purification materials are absent. Raising the temperature, as it is known, stimulates reactions with the formation of a volatile by-product and increases diffusion mobility of gas species through the column wall.

The second one is the super reactivity of as-milled solids. Powders produced by mechanical disintegration of solids in a cold state are oversaturated with defects. Activation barriers at this point abruptly decrease while the reactions and diffusion processes speed up. It should be also taken into consideration that reactive metals themselves already belong to the class of the most active chemical substances. That is, as-made powders of reactive metals and alloys are the best of what nature can offer us as purification material, of which all can be produced in the most economical manner. In fact, most of the above mentioned reactive metals are widely spread in the Earth crust, are easily available and are characterized by relatively low melting points.

Mechanochemical sorption apparatuses can be used not only for purification of flow gases [28] but also as vacuum getter pumps with controlled pumping speed, small weight and small energy consumption due to high fragility and low mechanical strength of the alloys [26]. This combination of characteristics makes the new getter pump attractable for employment in portable devices or in the measuring systems, where vibration is undesirable, e.g. in electronic microscopy.

One more application field for mechanochemical apparatuses is mechanochemistry itself, where our apparatuses are ready to become one of the instruments for research of mechanochemical reactions of gas/solid or liquid/solid type [27] [28]. Building up experimental dependences of $z-t$ type, where $z$ is the property under measurement and $t$ is the time interval between the milling of the ingot and the beginning of the measurement, will allow the separation of contributions from different driving forces of the process under study. This will improve our understanding of the nature of such reactions. A reactor with a transparent wall will give a chance to visualize a lot of changes in the state of the system under study and use this information either for research purposes or for demonstration purposes in teaching the basics of mechanochemistry.

The general view of the disintegrator used for the production of reactive powders, the sorption characteristics of which are given in Figure 2 and Figure 3, is shown in Figure 6.

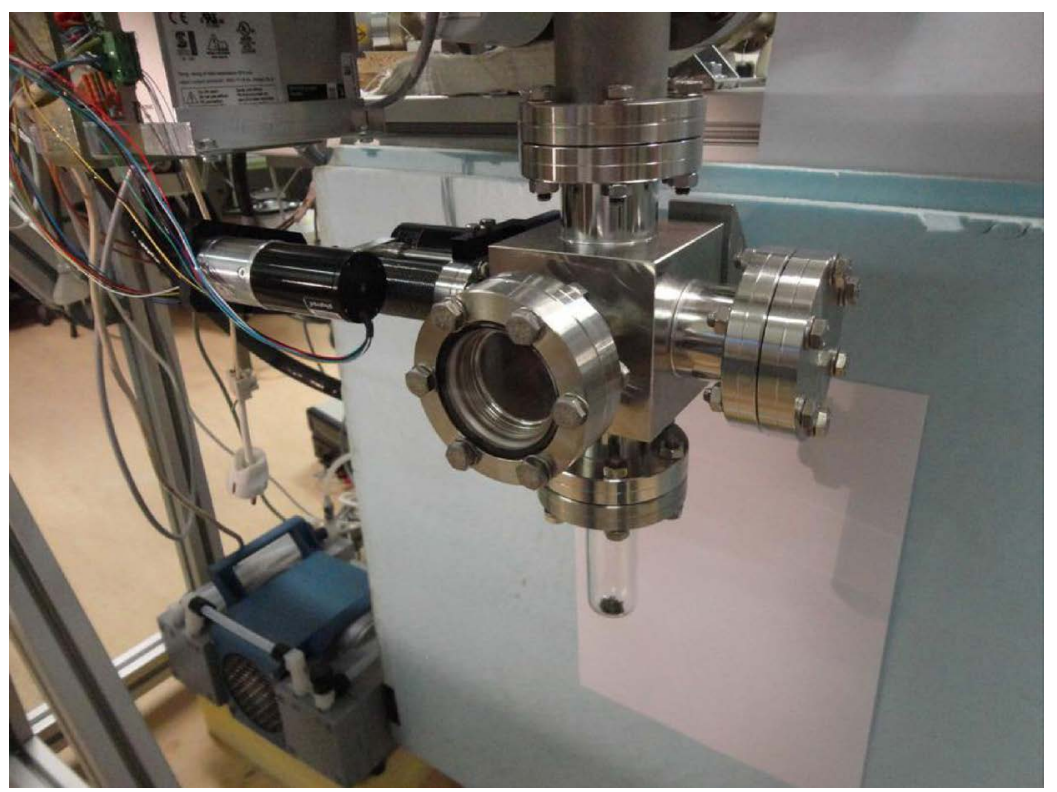

Figure 6. Vacuum milling chamber. 


\section{Sorption Columns of the Fluidized Bed Type}

The third type of gas purification equipment working on metal reactive powders is schematically shown in Figure 7 [29]. The sorption column 1 reaches maximally high efficiency due to fluidized bed technology: here the inlet of the gas to be purified is located not from above but from below upwards through valve 3 and filter 2 .

The regime of fluidized bed is set when the rate of the gas fed from below begins to exceed the incipient fluidization point [40]-[42]. From this moment the rising flows of gas $X+Y$ set the particles of Me, which were earlier resting on the distributor plate 4 , into chaotic movement. Colliding with each other, these particles release from the growing layer of products $\mathrm{MeY}$ on their surface, and this maintains the reaction $\mathrm{Me}+\mathrm{Y}=\mathrm{MeY}$ on a higher kinetic level than in gas purifiers of in-line type, where the rate is limited by the diffusion of the reagents through the layer of product MeY.

Small particles of MeY appearing as the result of chemical reactions and mechanical collisions in the fluidization zone are taken upwards by the gas flow, where the separation of the mixture $\mathrm{MeY}+\mathrm{X}$ takes place: molecules of gas $X$ passing through the exhaust filter 9 , the outside filter 8 , and the valve 7 , and then leave the sorption column. Solid particles MeY then go through the discharge port 6 into the receiver of solid waste 5 .

Sorption columns of the given type have the highest productive capacity compared to the previously discussed equipment and for this reason they are more suitable than the others for the mass production of pure and high purity gases. In contrast to the mechanochemical sorption apparatuses, where the reaction takes place in a thin layer of fresh powder, here the reaction involves the entire thickness of the material filled into the column. If the sorption columns are compared with the improved gas purifiers, they clearly show superiority in the process kinetics and are more convenient in industrial conditions because they do not need to be periodically replaced by replenished purifier components. Sorption columns (Figure 7), being the equipment of a stationary type, are replenished when necessary with fresh sorbent in their working position through the filling port 10 and the waste is separated from the system via the replaceable receiver 5 .

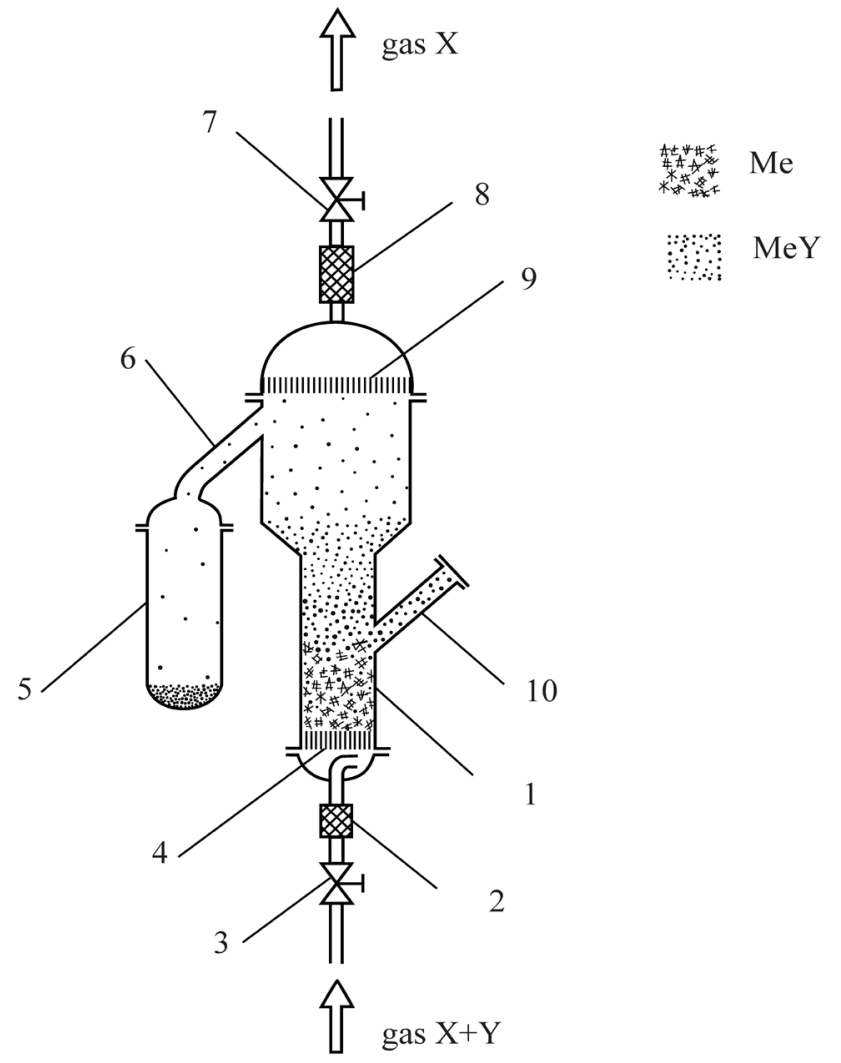

Figure 7. Sorption column of fluidized type: 1: a sorption column; 2: a filter; 3: a valve; 4: a distributer; 5: a receiver; 6 : discharge port; 7: a valve; 8: an outside filter; 9: an exhaust filter; 10: a filling port. 


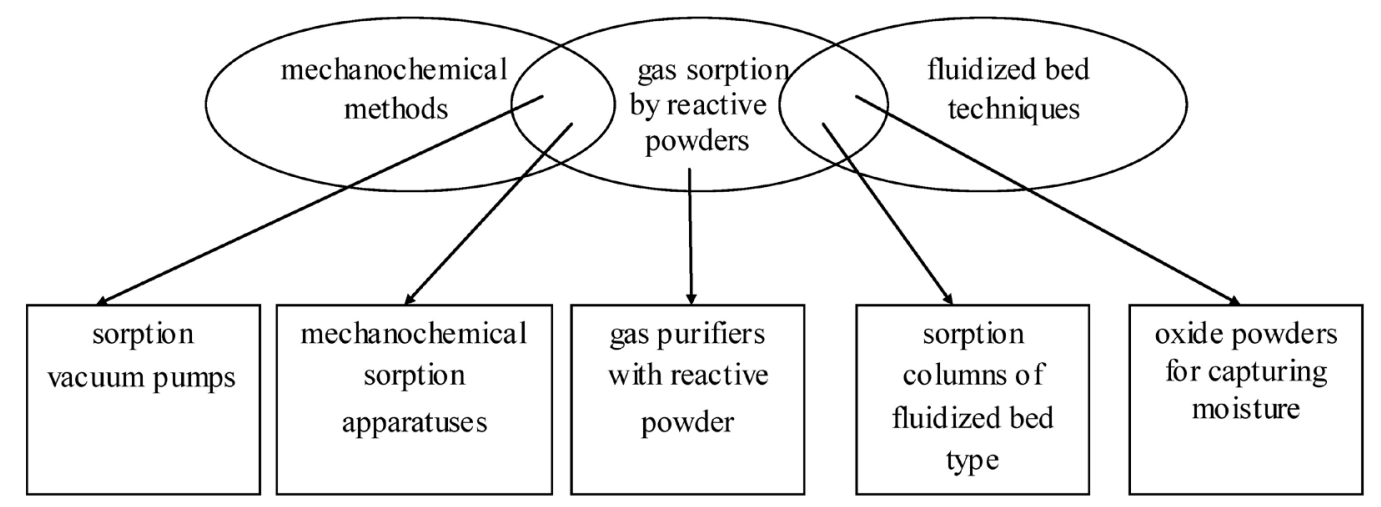

Figure 8. The structure of powder direction in getter technologies.

In addition to gas purification technologies, a sorption column of fluidized bed type can be used as a factory for the production of nano- or micro-particles of oxides of reactive metals, e.g. $\mathrm{BaO}$ or $\mathrm{CaO}$, which are the best dryer materials. For the production of these kind of powders it is enough to blow argon with a small concentration of oxygen from below through the layer of the initial metallic particles and then small oxide particles, which are formed in the fluidized zone, are lifted by gas flow upward and are accumulated in receiver 5, which in this case becomes a collector of the end product.

\section{Conclusions}

Sorption apparatuses and devices with reactive metal powder are the new technological interdisciplinary directions in getters and gas purification, which appeared from the intersection of three fields: sorption of gases by reactive metals, mechanochemistry, and fluidized bed technique (Figure 8). In its content this direction does not go beyond the framework of the known getter applications; however, it radically improves the circumstances involved in their use. This takes place due to the improved sorption capacities and the improved economies associated with reactive metals, technological innovations and construction techniques, which allowed the creation of new gas purification equipment. The new reality in the getter field can now be fully understood taking into account the following developments:

- improved gas purifiers using reactive powder, the cost of which is by 10 times lower and the sorption capacity of which is by 100 times higher than those of the prototype using getters of a different class;

- mechanochemical sorption apparatuses, which for the first time performed operating control of the sorption process and purification of flow gas by mechanical activation of a reagent in situ and in statu nascendi;

- sorption columns of fluidized type for mass production of pure and high purity gases or for the production of powder drying materials of the composition $\mathrm{BaO}$ or $\mathrm{CaO}$.

At present it is difficult to predict all the consequences of industrialization of these developments. Certainly a multiple increase in the life span of gas purification systems and decrease in their size will take place; gases of higher purity grade than the purity grade achieved for today will become available with a much lower cost stack than for the current product. With that said, however, the authors realize that despite the simplicity and obviousness of the suggested solutions it will take time until they are accepted by specialists in these fields, as this is connected with break of paradigms in the field, where nothing has essentially changed for a long time.

\section{References}

[1] Ferrario, B. (1996) Chemical Pumping in Vacuum Technology. Vacuum, 47, 363-370. http://dx.doi.org/10.1016/0042-207X(95)00252-9

[2] Chuntonov, K. and Yatsenko, S. (2013) Getter Films for Small Vacuum Chambers. Recent Patent on Materials Science, Bentham Science Publishers, 6, 29-39. http://dx.doi.org/10.2174/1874464811306010029

[3] Fransen, J.J.B. and Perdijk, H.J.R. (1960) The Absorption of Gases by Barium Getter Films Applied as a Tool. Vacuum, 10, 199-203. http://dx.doi.org/10.1016/0042-207X(60)90136-6

[4] Ricca, F. and della Porta, P. (1960) Carbon Monoxide Sorption by Barium Films. Vacuum, 10, 215-222.

http://dx.doi.org/10.1016/0042-207X(60)90140-8 
[5] della Porta, P. and Argano, E. (1960) Nitrogen Sorption by Barium Films. Vacuum, 10, 223-226. http://dx.doi.org/10.1016/0042-207X(60)90141-X

[6] Turnbull, J.C. (1977) Barium, Strontium, and Calcium as Getter in Electron Tubes. Journal of Vacuum Science \& Technology A, 14, 636-639. http://dx.doi.org/10.1116/1.569166

[7] Verhoeven, J. and van Doveren, H. (1982) Interaction of Residual Gases with Barium Getter Film as Measured by AES and XPS. Journal of Vacuum Science \& Technology, 20, 64-74. http://dx.doi.org/10.1116/1.571310

[8] Sparks, D.R. (2005) Method of Forming a Reactive Material and Article Formed thereby. US Patent 6923625.

[9] Silvernail, J.A. (2006) Protected Organic Electronic Device Structures Incorporating Pressure Sensitive Adhesive and Desiccant. US Patent No. 6998648.

[10] Chuntonov, K. and Setina, J. (2008) New Lithium Gas Sorbents: I. The Evaporable Variant. Journal of Alloys and Compounds, 455, 489-496. http://dx.doi.org/10.1016/j.jallcom.2007.01.158

[11] Chuntonov, K., Setina, J., Ivanov, A. and Permikin, D. (2008) New Lithium Gas Sorbent: III. Experimental Data on Evaporation. Journal of Alloys and Compounds, 460, 357-362. http://dx.doi.org/10.1016/j.jallcom.2007.06.055

[12] Chuntonov, K. (2011) Lithium or Barium Based Film Getters. US Patent Application 20110217491.

[13] Horie, H., Fukuda, Y., Kato H., Nakashima, N. and Makino, Y. (2011) Getter Material and Evaporable Getter Device Using the Same, and Electron Tube. US Patent No. 7927167.

[14] Giedraitis, A., Tamulevicius, S., Gudaitis, R. and Andrulevicius, M. (2010) Kinetics of Growth and Sorption Properties of Evaporable Barium Getter Films. Materials Science (Medziagotyra), 16, 12-23.

[15] Alvarez Jr., D. (2001) Method and Apparatus for Purification of Hydride Gas Streams. US Patent No. 6241955.

[16] Londer, H., Myneni, G.R., Adderley, P., Bartlok, G., Knapp, W., Schleussner, D. and Ogris, E. (2007) New High Capacity Getter for Vacuum Insulated Mobile $\mathrm{LH}_{2}$ Tank Systems. Vacuum, 82, 431-434. http://dx.doi.org/10.1016/j.vacuum.2007.07.063

[17] Sparks, D.R., Najafi, N. and Newman, B.E. (2010) Getter Device. US Patent No. 7789949.

[18] Giannantonio, R., Vescovi, C., Cattaneo, L. and Longoni, G. (2011) Getter Systems Comprising an Active Phase Inserted in a Porous Material Distributed in a Low Permeability Means. US Patent No. 7977277.

[19] Giannantonio, R., Longoni, G., Vescovi, C. and Cattaneo, L. (2014) Getter System Comprising One or More Deposits of Getter Material and a Layer of Material for the Transport of Water. US Patent No. 8911862.

[20] Chuntonov, K. (2013) Safe Gas Sorbents with High Sorption Capacity on the Basis of Lithium Alloys. US Patent No. 8529673.

[21] Chuntonov, K. (2014) Barium Containing Granules for Sorption Applications. US Patent No. 8623302.

[22] Chuntonov, K. (2014) Apparatus and Method for Droplet Casting of Reactive Alloys and Applications. US Patent Application 20140290897.

[23] Boffito, C. and Schiabel, A. (1994) Process for the Sorption of Residual Gas by Means of a Non-Evaporated Barium Getter Alloy. US Patent No. 5312606.

[24] Schiabel, A. and Boffito, C. (1994) Process for the Sorption of Residual Gas by Means of a Non-Evaporated Barium Getter Alloy. US Patent No. 5312607.

[25] Manini, P. and Belloni, F. (1997) Device for Maintaining a Vacuum in a Thermally Insulating Jacket and Method of Making Such Device. US Patent No. 5600957.

[26] Chuntonov, K. (2013) Sorption Pump with Mechanical Activation of Getter Material and Process for Capturing of Active Gases. US Patent Application 2013078113.

[27] Chuntonov, K. and Lee, M.K. (2014) Mechanochemical Sorption Apparatuses. Advanced Materials Research, 875-877, 1106-1110. http://dx.doi.org/10.4028/www.scientific.net/AMR.875-877.1106

[28] Chuntonov, K. (2015) Sorption Apparatus for the Production of Pure Gases. US Patent No. 9095805.

[29] Chuntonov, K. and Setina, J. (2015) Activationless Gas Purifiers with High Sorption Capacity. US Patent Application (Patent Pending).

[30] Kubaschewski, O. and Hopkins, B.E. (1962) Oxidation of Metals and Alloys. Butterworths, London.

[31] Hauffe, K. (1966) Reaktionen in und an festen Stoffen. 2. Auflagen, Springer-Verlag, Berlin. http://dx.doi.org/10.1007/978-3-642-88042-1

[32] Meyer, K. (1968) Physikalisch-chemische Kristallographie. VEB Deutscher Verlag, Leipzig.

[33] Fromm, E. and Gebhardt, E. (1976) Gase und Kohlenstoff in Metallen. Springer-Verlag, Berlin. http://dx.doi.org/10.1007/978-3-642-80943-9 
[34] della Porta, P. (1992) “Gettering”: An Integral Part of Vacuum Technology. Proceedings of the 39th National Symposium of American Vacuum Society, Chicago, 9-13 November 1992, Technical Paper TP 202.

[35] Giorgi, E. and Ferrario, B. (1989) High-Porosity Thick-Film Getters. IEEE Transactions on Electron Devices, 36, 2744-2747. http://dx.doi.org/10.1109/16.43783

[36] ASTM F 798-97 (2002) Standard Practice for Determining Gettering Rate, Sorption Capacity, and Gas Content of Non-Evaporable Getters in the Molecular Flow Region.

[37] Erjavec, B. and Setina, J. (2011) Investigations of a Method for Determining Pumping Speed and Sorption Capacity of Nonevaporable Getters Based on in Situ Calibrated Throughput. Journal of Vacuum Science \& Technology A, 29, Article ID: 051602. http://dx.doi.org/10.1116/1.3626535

[38] Brochure SAES Getters (2004) Solution for Flat Panel Displays.

[39] Toia, L. and Boffito, C. (2003) Non-Evaporable Getter Alloys. US Patent No. 6521014.

[40] Gupta, C.K. and Sathiyamoorthy, D. (1999) Fluid Bed Technology in Materials Processing. CRC Press LLC, Boca Raton.

[41] Zhu, J., Leckner, B., Cheng, Y. and Grace, J.R. (2005) Fluidized Beds. In: Crowe, C.T., Ed., Multiphase Flow Handbook, CRC Press, Boca Raton, 5-1-5-93. http://dx.doi.org/10.1201/9781420040470.ch5

[42] Epstein, N. and Grace, J.R. (2010) Spouted and Spout-Fluid Beds: Fundamentals and Applications. Cambridge University Press, Cambridge. http://dx.doi.org/10.1017/CBO9780511777936 